Revista Internacional de Ciencias Podológicas

ISSN: 1989-5151

\title{
Valor del Test de Silfverskiöld para el diagnóstico de la fascitis plantar
}

\author{
José-Antonio García Vidal', José-Gregorio Piñero Palazón², Aitor Baño Alcaraz ${ }^{3}$, María-Piedad Sánchez \\ Martínez $z^{4}$ Francesc Medina i Mirapeix ${ }^{5}$
}

Fecha de recepción: 4 de abril de 2018 / Fecha de aceptación: 11 de septiembre de 2018

Resumen. Introducción: La fascitis plantar (FP) está descrita como la causa más común de dolor en el talón. Aunque se considera una patología multifactorial, la limitación de la dorsiflexión del tobillo es uno de los factores más determinantes, predisponiendo al desarrollo de patologías en el miembro inferior.

Objetivo: Determinar la exactitud del test de Silfverskiöld como herramienta diagnóstica de la FP a partir del análisis de varios indicadores (sensibilidad, especifidad, valor predictivo positivo y negativo, y razones de verosimilitud positiva y negativa).

Método: Se realizó un estudio de validez transversal en 16 sujetos ( 9 mujeres y 7 hombres) diagnosticados de fascitis plantar unilateral de al menos 3 meses de evolución. Un fisioterapeuta se encargó de evaluar el déficit de dorsiflexión en ambos tobillos mediante el Test de Silfverskiöld.

Resultados: Fueron descartados aquellos pies sin problemas de movilidad en dorsiflexión de tobillo, por lo que finalmente fueron analizados 14 pies afectos y 8 sanos. los indicadores de eficacia diagnóstica del test de Silfverskiöld en la FP. Al determinar la eficacia del test de Silfverskiöld como predictor de FP encontramos una sensibilidad de $93,7 \%$, especificidad del 75\%, valor predictivo positivo (VPP) de 86,6\% y valor predictivo negativo (VPN) de $85,7 \%$. La razón de verosimilitud positiva (RV+) fue de 3,74, mientras que la negativa (RV-) resultó 12,5.

Conclusión: El test de Silfverskiöld puede ser considerado una prueba eficaz para el diagnóstico de la FP. Existe una importante asociación entre la limitación en la dorsiflexión de tobillo por restricción de gemelos y la probabilidad de padecer FP.

Palabras clave: Fascitis plantar; gastrocnemios; test de Silfverskiöld.

\section{[en] Value of the Silfverskiöld Test for the diagnosis of plantar fasciitis}

Abstract. Background: Plantar fasciitis (PF) is described as the most common cause of heel pain. Although it is considered a multifactorial pathology, the limitation of dorsiflexion of the ankle is one of the most determining factors, predisposing to the development of pathologies in the lower limb.

Objective: To determine the accuracy of the Silfverskiöld test as a diagnostic tool for PF, based on the analysis of several indicators (sensitivity, specificity, positive and negative predictive value, and positive and negative likelihood ratios).

Method: A cross-sectional validity study was conducted in 16 subjects ( 9 women and 7 men) diagnosed with unilateral plantar fasciitis of at least 3 months evolution. A physiotherapist was responsible for assessing the deficit of dorsiflexion in both ankles by the Silfverskiöld test.

Outcome: Those feet without mobility problems in ankle dorsiflexion were discarded, so finally 14 affected feet and 8 healthy ones were analyzed. the indicators of diagnostic efficacy of the Silfverskiöld test in PF. When determining the efficacy of the Silfverskiöld test as a predictor of PF, we found a sensitivity of $93.7 \%$, specificity of $75 \%$, positive predictive value (PPV) of $86.6 \%$ and negative predictive value (NPV) of $85.7 \%$. The positive likelihood ratio (LR+) was 3.74, while the negative (LR-) ratio was 12.5. Conclusion: The Silfverskiöld test can be considered an effective test for the diagnosis of PF. There is an important association between limitation in dorsiflexion of the ankle due to restriction of twins and the probability of suffering PF.

Keywords: Plantar fasciitis; gastrocnemius; Silfverskiöld test.

1 Diplomado en Fisioterapia. Doctor por la Universidad de Murcia. Profesor del Departamento de Fisioterapia de la Universidad de Murcia. garciavidal@um.es

2 Diplomado en Fisioterapia.

josegregorio.pinero22@gmail.com

3 Diplomado en Fisioterapia. Doctor por la Universidad de Sevilla. Profesor del Departamento de Fisioterapia de la Universidad de Murcia. aitor.bano@um.es

4 Diplomado en Fisioterapia. Doctor por la Universidad de Murcia. Catedrático de Escuela Universitaria. Departamento de Fisioterapia de la Universidad de Murcia.

mariapiedadsanchez@hotmail.com

5 mirapeix@um.es 
Sumario: 1. Introducción. 2. Objetivos. 3. Material y métodos. 4. Discusión. 5. Limitaciones. 6. Conclusiones. 7. Bibliografía.

Cómo citar: García Vidal, J. A.; Piñero Palazón, J. G.; Baño Alcaraz, A. Sánchez Martínez, M. P.; Medina i Mirapeix, F. (2018). Valor del Test de Silfverskiöld para el diagnóstico de la fascitis plantar, en Revista internacional de ciencias podológicas 13 (1), 41-46.

\section{Introducción}

La fascitis plantar (FP) es un proceso crónico no inflamatorio que cursa con engrosamiento y degeneración mixoide de la fascia plantar, normalmente en su zona insercional a nivel del calcaneo $^{2}$. Los síntomas vinculados a ella son la causa más común de dolor en el talón, afectando aproximadamente al $10 \%$ de la población en algún momento de su vida. ${ }^{1-3}$ Se estima que se producen alrededor de un millón de visitas médicas anuales en EEUU diagnosticadas como FP. ${ }^{1-4}$ En este mismo país constituye entre el $11 \%$ y el $15 \%$ de todas las visitas a profesionales médicos por dolor en el pie. ${ }^{5} \mathrm{La}$ edad media de presentación suele hallarse en la sexta década de vida, siendo más frecuente en mujeres ${ }^{6,7}$, aunque este pico de edad es menor en la población deportista. ${ }^{8}$

Los principales síntomas de la FP son dolor y dificultad para caminar, siendo particularmente severo en los primeros pasos de la mañana o después de un periodo de descanso. Ocasionalmente el dolor puede extenderse a todo el pie incluyendo los dedos. Como consecuencia del impacto que tiene en la calidad de vida y de su potencial tendencia natural a convertirse en una patología crónica y recidivante (con periodos fluctuantes de mayor y menor sintomatología), la condición representa una carga económica importante para los servicios de salud. ${ }^{3,9}$

A pesar de la alta prevalencia, en un $85 \%$ de los casos el origen no ha sido determinado.0 El diagnóstico de esta patología suele ser clínico en base a los síntomas, debiéndose confirmar con una ecografía. Los hallazgos más frecuentes suelen ser aumento del grosor, hipoecogenicidad y biconvexidad de la inserción. En ocasiones también pueden observarse ruptura parcial, edema perifascial y calcificación intratendinosa. Rara vez se utilizan medios como la gammagrafía o la RMN. ${ }^{11}$ Las radiografías simples del pie suelen ser poco útiles, ya que no existe una correlación clínico-radiológica: hasta el 15$20 \%$ de las personas con espolón calcáneo en una radiografía no tienen dolor plantar, y sólo el 5\% de los pacientes con dolor plantar tienen un espolón en la radiografía. ${ }^{12}$

Se ha mostrado en cadáveres que existe una continuidad de la fascia plantar con el paratendón del tendón de Aquiles, proporcionando una correlación positiva entre la carga del tendón de Aquiles y la tensión de la fascia plantar. ${ }^{13}$ Esta ampliamente aceptado que la disminución de la dorsiflexión de tobillo es uno de los principales factores de riesgo para desarrollar tanto fascitis ${ }^{14-18}$ como otras patologías del miembro inferior (tendinopatía Aquílea ${ }^{19-21}$, esguince de tobillo, atrapamiento nervioso en el dorso del pie y metatarsalgia ${ }^{22-23}$, lesiones del ligamento cruzado anterior ${ }^{24}$, tendinopatía rotuliana ${ }^{25}$, síndrome de dolor patelofemoral ${ }^{21,23,26}$ y Morbus Osgood Schlatter ${ }^{27}$ ).

La disminución de la movilidad del tobillo puede ser debida a restricción de tejidos musculares biarticulares (ej. Gemelo) o a monoarticulares (ej. Soleo) o bien a problemas articulares. Para discriminar groseramente si la limitación es debida a una u otra causa, tradicionalmente se ha utilizado test de Silfverskiöld, el cual compara las limitaciones de dorsiflexión de tobillo existentes al explorar ésta en dos posiciones de prueba, con la rodilla extendida y ligeramente flexionada. Para tal fin, este test ha mostrado buena validez. ${ }^{15}$ Sin embargo, a nuestro conocimiento aún no se ha utilizado los resultados del test para complementar el diagnóstico de la FP.

El objetivo de estudio fue determinar la exactitud del test de Silfverskiöld como herramienta diagnóstica de la FP a partir del análisis de varios indicadores (sensibilidad, especifidad, valor predictivo positivo y negativo, y razones de verosimilitud positiva y negativa). Nuestra hipótesis fue que los sujetos con limitación en la dorsiflexión de tobillo asociada a restricción de gemelos (aquellos en el test de Silfverskiöld muestran limitación de la dorsiflexión del tobillo en la posición de rodilla extendida pero no en la de rodilla flexionada) tienen mayor probabilidad de tener FP. 


\section{Métodos}

Se realizó un estudio de validez transversal entre noviembre de 2016 y mayo de 2017 por la Universidad de Murcia y el Hospital General Universitario Reina Sofía de la Región de Murcia. Los participantes se seleccionaron entre pacientes del hospital que cumplieron los siguientes criterios de inclusión: mayor de 18 años, con FP unilateral diagnosticada con al menos 3 meses de evolución. Los criterios de exclusión fueron con dificultad en el idioma, intervención quirúrgica en esa zona, tratamiento con corticoides en los 6 meses previos y FP bilateral. Los pacientes fueron captados en consulta por su médico y un investigador comprobó que cumplía los criterios de elegibilidad. En caso de cumplir todos los criterios se les explicaba el estudio y se suministraba el consentimiento informado, el cual era firmado por los pacientes. A continuación se procedía a la medición y realización del test.

Las variables evaluadas en la investigación fueron: sociodemográficas (edad y sexo), estructurales (peso, altura e índice de masa corporal) y tiempo de evolución de la patología mediante entrevista, y por medio de examen físico en decúbito supino se realizó el test de Silfverskiöld en ambos MMII. Las impresiones clínicas fueron medidas y registradas por un examinador experto.

El Test de Silfverskiöld es una prueba que explora en decúbito supino el grado de dorsiflexión del tobillo en dos posiciones de prueba: con rodilla extendida y flexionada. En ambas, la exploración se realiza con las articulaciones del retropié mantenidas en una posición reducida, para bloquearlas y evitar errores de medición. El test compara los resultado alcanzados en ambas posiciones, y como describieron Patel et al en 2011, considera que el test es positivo "para la restricción aislada de gemelos" cuando aparece una limitación importante de la dorsiflexión en extensión de rodilla, que se corrige con flexión de la rodilla a 90 grados. Según esos autores, el test también se puede considerar positivo "para la restricción del complejo gastro-sóleo" o por un problema articuar cuando se presenta una limitación de la dorsiflexión tanto con rodilla extendida como flexionada. ${ }^{15}$

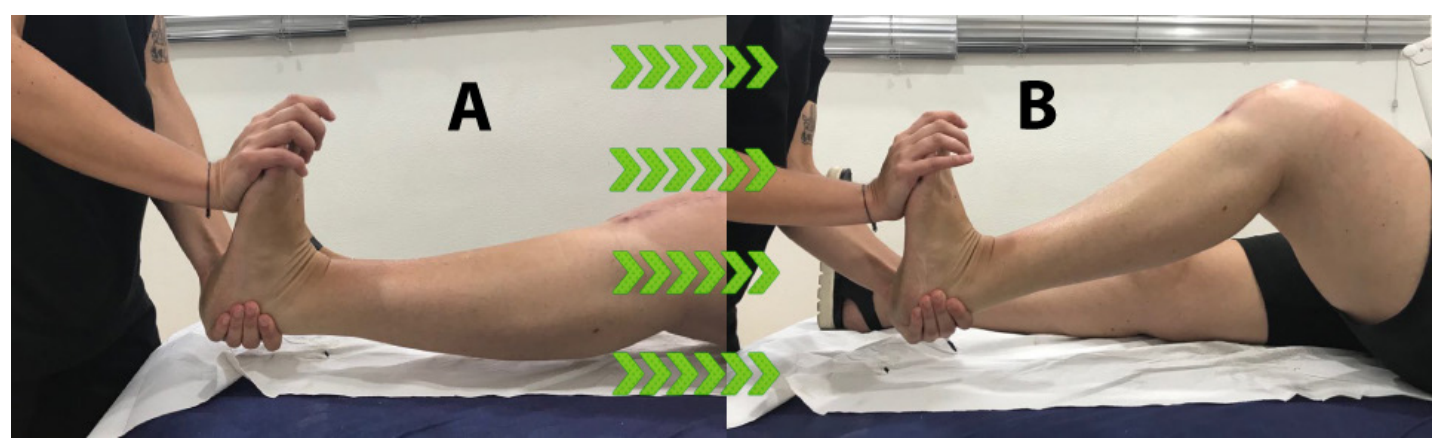

Figura 1. Test de Silfverskiöld: dorsiflexión de tobillo con extensión de rodilla (A) y con flexión de rodilla (B).

\subsection{Análisis estadístico}

El análisis de los datos se realizó mediante el programa estadístico IBM SPSS (SPSS v.23; IBM SPSS, Chicago, Illinois). Se describió los participantes utilizando rangos, medias y desviaciones típicas (DT) para las variables cuantitativas y frecuencias (n) y porcentajes (\%) para las cualitativas. Las unidades de estudio (los pies) se clasificaron en afecto/ no afecto de FP, y con o sin limitación de movilidad en una o ambas posiciones del Test de Silfverskiöld. Para los análisis encaminados a determinar la exactitud del test se incluyeron exclusivamente las unidades de estudio que tuvieron algún tipo de limitación de la movilidad, ya fuera un pie afecto o no. Los indicadores de exactitud diagnóstica establecidos fueron sensibilidad, especificidad, valor predictivo positivo $\mathrm{y}$ negativo, razón de verosimilitud positiva y razón de verosimilitud negativa.

\section{Resultados}

La muestra del estudio finalmente contó con 16 sujetos diagnosticados con FP, Concreta- 
mente la muestra está formada por 9 mujeres que comprenden el $56^{\prime} 3 \%$ de la muestra y 7 hombres que configuran el restante $43,8 \%$. Los sujetos presentaban un rango de edad entre 35 y 68 años, con una edad media \pm D.E. de $53^{\prime} 75 \pm$ 9'92.
Se presentó una dominancia de un $93{ }^{\prime} 8 \%$ para el MI derecho y un 6'3\% de dominancia en ambos miembros inferiores. El promedio del IMC fue $28^{\prime} 99 \mathrm{~kg} / \mathrm{m}^{2}$ (DT=4'26), por lo que se trata de una población con sobrepeso grado II. (Tabla 1).

Tabla 1. Características de la población de estudio

\begin{tabular}{|l|l|c|c|}
\hline \multicolumn{2}{|c|}{ Variables } & Media ó n & DT ó \% \\
\hline Edad (años) & & 53,75 & 9,92 \\
\hline \multirow{2}{*}{ Sexo } & Hombre & 7 & 43,8 \\
\cline { 2 - 4 } & Mujer & 9 & 56,3 \\
\hline \multirow{2}{*}{ IMC $\left(\mathrm{kg} / \mathrm{m}^{-2}\right)$} & & 28,99 & 4,26 \\
\hline \multirow{2}{*}{ Dominancia MMII } & Diestro & 15 & 93,8 \\
\cline { 2 - 4 } & Bilateral & 1 & 6,3 \\
\hline
\end{tabular}

De los 16 pies con FP, 2 (12,5\%) no tuvieron problemas de movilidad en la dorsiflexión de tobillo y no fueron incluidos en el análisis de la exactitud diagnóstica. De los 16 pies sanos y sin $\mathrm{FP}$, sólo 8 presentaron restricción de movilidad. Así, finalmente fueron analizados 14 pies afectos y 8 sanos. En la Tabla 2 se puede observar que 13 de los 16 pies afectos ofrecieron un test de Silfverskiöld positivo "para la restricción de gemelos", y en cambio solo 2 de los 8 pies sanos.

Tabla 2. Eficacia del test de Silfverskiöld en el diagnóstico de la FP

\begin{tabular}{|l|r|r|r|}
\hline \multirow{2}{*}{} & \multicolumn{2}{|c|}{ Fascitis Plantar } & \multirow{2}{*}{ Total } \\
\cline { 2 - 3 } & $\mathrm{Si}$ & No & \\
\hline Positivo ("para restricción de gemelos") & 13 & 2 & 15 \\
\hline Negativo & 1 & 6 & 7 \\
\hline Total & 14 & 8 & 22 \\
\hline
\end{tabular}

La Tabla 3 muestra los indicadores de eficacia diagnóstica del test de Silfverskiöld en la FP. Al determinar la eficacia del test de Silfverskiöld como predictor de FP encontramos una sensibilidad de 93,7\%, especificidad del 75\%, valor predictivo positivo (VPP) de $86,6 \%$ y valor predictivo negativo (VPN) de $85,7 \%$. La razón de verosimilitud positiva $(\mathrm{RV}+)$ fue de 3,74 , mientras que la negativa $(\mathrm{RV}-)$ resultó 12,5 .

Tabla 3. Indicadores de eficacia del test de Silfverskiöld en el diagnóstico de la FP

\begin{tabular}{|l|c|}
\hline & Test de Silfverskiöld \\
\hline Sensibilidad & $\mathbf{9 3 , 7 \%}$ \\
\hline Especificidad & $\mathbf{7 5 \%}$ \\
\hline Valor Predictivo Positivo (VPP) & $\mathbf{8 6 , 6 \%}$ \\
\hline Valor Predictivo Negativo (VPN) & $\mathbf{8 5 , 7 \%}$ \\
\hline Razón de Verosimilitud Positiva (RV+) & $\mathbf{3 , 7 4}$ \\
\hline Razón de Verosimilitud Negativa (RV-) & $\mathbf{1 2 , 5}$ \\
\hline
\end{tabular}

\section{Discusión}

Hasta ahora, el test de Silfverskiöld había sido usado fundamentalmente como prueba ortopédi- ca para evaluar la retracción del complejo gastrosóleo. Extrapolado a la práctica traumatológica, los pacientes con FP, test positivo y mala respuesta al tratamiento conservador habían sido consi- 
derados candidatos a cirugía de liberación de la fascia plantar. Sin embargo, está demostrado que estas técnicas quirúrgicas clásicas producen una tasa de satisfacción inferior al 50\% debido a la persistencia de dolor y limitación. ${ }^{28}$ Es por esto que muchos autores optan por la liberación proximal del gemelo medial por mínima incisión ${ }^{30} \mathrm{u}$ otras similares (procedimiento de Strayer) como técnicas quirúrgicas de elección.

Aunque existen en la literatura diferentes procedimientos de ensayo para realizar el test, realizamos una interpretación del mismo siguiendo los criterios de Patel et al y de Baumbach et al. ${ }^{15,31}$ Nuestros resultados muestran que un elevado número de los miembros inferiores afectos con FP cuentan con una retracción aislada de gemelos, siendo el test de Silfverskiöld un método sensible $(93,7 \%)$ y específico $(75 \%)$ para su detección.

Se estima que al menos un $20 \%$ de la población sana padece acortamiento de gastrocne$\operatorname{mios}^{29}$. Según la mayoría de autores consultados, este hecho puede ser considerado como un factor predisponente para el desarrollo de pato- logías como la FP. Por ello consideramos que la detección precoz de estas retracciones proporciona una información relevante que puede ser utilizada por médicos y fisioterapeutas para mejorar los tratamientos, tanto conservadores como quirúrgicos. Coincidimos con algunos autores que afirman que esta prueba exploratoria puede servir para focalizar protocolos de terapia física más específicos, enfocando parte del tratamiento sobre los gemelos y no solo sobre la fascia plantar. ${ }^{15}$

Sería recomendable añadir este test a la exploración física convencional de miembros inferiores, tanto en pacientes con patología instaurada como de forma profiláctica en el resto.

\section{Conclusiones}

El test de Silfverskiöld puede ser considerado una prueba eficaz para el diagnóstico de la FP. Nuestro estudio indica que los sujetos con limitación en la dorsiflexión de tobillo asociada a restricción de gemelos tienen mayor probabilidad de padecer FP.

\section{Bibliografía}

1. Grieve R, Palmer S. Physiotherapy for plantar fasciitis: a UK-wide survey of current practice. Physiotherapy. 2016.

2. Lemont H, Ammirati K, Usen N. Plantar fasciitis: diagnosis and therapeutic considerations. Alternative medicine review 2005, 10 (2), 83-93.

3. Beeson P. Plantar fasciopathy: revisiting the risk factors. Foot Ankle Surg. 2014;20 (3):160-5.

4. Cutts S, Obi N, Pasapula C, Chan W. Plantar fasciitis. Ann R Coll Surg Engl. 2012;94 (8):539-42.

5. Arslan A, Koca TT, Utkan A, Sevimli R, Akel I. Treatment of Chronic Plantar Heel Pain With Radiofrequency Neural Ablation of the First Branch of the Lateral Plantar Nerve and Medial Calcaneal Nerve Branches. J Foot Ankle Surg. 2016;55 (4):767-71.

6. Rose B, Singh D. Inferior heel pain. Orthopaedics and Trauma. 2016;30 (1):18-23.

7. Artidiello Bustio D, Hernández Echevarría DC, Aguilar Artidiello H, Salazar Camacho MC. Fascitis plantar. Revista de Ciencias Médicas de Pinar del Río. 2015;19 (2):206-13.

8. Chana Valero P. Eficacia de los impulsos eléctricos de bajo voltaje en la fasciosis plantar: Universidad Complutense de Madrid; 2013.

9. Santa Coloma E, Khoury MA. Medicina basada en la evidencia: evidencia en el manejo no quirúrgico de la fascitis plantar. Rev Asoc Argent Traumatol Deporte. 2011;18 (2):81-9.

10. Yañez Arauz JM, Del Vecchio JJ, Raimondi N, Codesido MA. Riesgo de lesiones quirúrgicas en la fasciotomía plantar percutánea: estudio anatómico en cadáveres frescos. Revista de la Asociación Argentina de Ortopedia y Traumatología. 2011;76 (2):141-5.

11. Puttaswamaiah, R, Chandran, P. Degenerative plantar fasciitis: a review of current concepts. The Foot. 2007, $17(1), 3-9$.

12. Cornwall MW, MCPoil TG. Plantar fasciitis: Etiology and treatment. J Orthop Sports Phys Ther. 1999;29:756-60.

13. Martinelli N, Bonifacini C, Romeo G. Current therapeutic approaches for plantar fasciitis. Orthop Res Rev. 2014;6:33-40. 
14. Lim AT, How CH, Tan B. Management of plantar fasciitis in the outpatient setting. Singapore Med J. 2016;57 (4):168-70.

15. Patel A, DiGiovanni B. Association between plantar fasciitis and isolated contracture of the gastrocnemius. Foot \& ankle international. 2011;32 (1):5-8.

16. Barry M. Causation and risk factors of Plantar Fasciitis. Acc Research. 2016;12 (1):1-37.

17. League AC. Current concepts review: plantar fasciitis. Foot \& ankle international. 2008;29 (3):358-66.

18. Zhang X, O'Meara D, Vanwanseele B, Hunt A, Smith R, editors. The influence of heel height on ankle kinematics during standing, walking, jogging and sidestepping in children. In: ISBS-Conference Proceedings Archive: 33rd Internacional Conference on Biomechanics in Sports. France; 2015. p. 7536.

19. Whitting JW, Steele JR, Mcghee DE, Munro BJ. Dorsiflexion capacity affects achilles tendon loading during drop landings. Medicine and science in sports and exercise. 2011;43 (4):706-13.

20. Pope R, Herbert R, Kirwan J. Effects of ankle dorsiflexion range and pre-exercise calf muscle stretching on injury risk in Army recruits. Australian Journal of Physiotherapy. 1998;44 (3):165-72.

21. Rabin A, Kozol Z, Finestone AS. Limited ankle dorsiflexion increases the risk for mid-portion Achilles tendinopathy in infantry recruits: a prospective cohort study. Journal of foot and ankle research. 2014;7 (1):48-22. Riddle DL, Pulisic M, Pidcoe P, Johnson RE. Risk factors for plantar fasciitis: a matched case-control study. J Bone Joint Surg Am. 2003;85 (5):872-7.

23. Barrett SL, Jarvis J. Equinus deformity as a factor in forefoot nerve entrapment: treatment with endoscopic gastrocnemius recession. Journal of the American Podiatric Medical Association. 2005;95 (5):464-

24. Wahlstedt C, Rasmussen-Barr E. Anterior cruciate ligament injury and ankle dorsiflexion. Knee Surgery, Sports Traumatology, Arthroscopy. 2015;23 (11):3202-7.

25. Backman LJ, Danielson P. Low range of ankle dorsiflexion predisposes for Patellar Tendinopathy in Junior Elite Basketball Players a 1-Year prospective study. The American journal of sports medicine. 2011;39 (12):2626-33.

26. Piva SR, Goodnite EA, Childs JD. Strength around the hip and flexibility of soft tissues in individuals with and without patellofemoral pain syndrome. Journal of orthopaedic \& sports physical therapy. 2005;35 (12):793-801.

27. Šarčević Z. Limited ankle dorsiflexion: a predisposing factor to Morbus Osgood Schlatter?. Knee Surgery, Sports Traumatology, Arthroscopy. 2008;16 (8):726-8.

28. Davies MS, Weiss GA, Saxby TS. Plantar fasciitis: how successful is surgical intervention? Foot \& Ankle International. 1999;20 (12):803-7.

29. Barouk LS, Barouk P. Gastrocnemios cortos. Revista del Pie y Tobillo, 2012;26 (2), 7-13.

30. De los Santos Real R, Muñoz PM, Rodríguez JP, Alonso JE. Liberación proximal del gemelo medial por mínima incisión. Revista del Pie y Tobillo. 2011;25 (1):42-6.

31. Baumbach SF, Braunstein M, Seeliger F, Borgmann L, Böcker W, Polzer H. Ankle dorsiflexion: what is normal? Development of a decision pathway for diagnosing impaired ankle dorsiflexion and M. gastrocnemius tightness. Archives of Orthopaedic and Trauma Surgery. 2016;136 (9):1203-1 